\title{
Optimum design and research on novel vehicle hybrid excitation synchronous generator
}

\author{
Zhong-Shu Liu * \\ The Key Laboratory for Automotive Electronics and Electric Drive of Fujian Province /School of Information Science and Engineering, \\ Fujian University of Technology, Minhou, Fuzhou City, 350118, Fujian, China
}

\begin{abstract}
Hybrid excitation is an organic combination of permanent magnet excitation and electric excitation. Hybrid excitation synchronous generator (HESG) both has the advantages of light quality, less losses and high efficiency like permanent magnet generator and the advantages of good magnetic field adjusting performance like electric excitation generator, so it is very suitable for the vehicle application. This paper presented a novel vehicle HESG which has skew stator core, permanent magnet rotor and both armature winding and field winding in the stator. Using ANSYS software, simulating the electric excitation field and the magnetic field, and finally the main parameters of HESG were designed. The simulation and the test results both show that the novel vehicle PMSG has the advantages of small cogging torque, high efficiency, small harmonic component output voltage and low waveform aberration, so as to meet the design requirements fully.
\end{abstract}

\section{Introduction}

With the rapid development of national economy, automobile industry has become one of the pillar industries of China economy, it plays an irreplaceable role in all aspects of transportation and human daily life. Generator is the key to the car electrical system components, the progressiveness and comfort of the car are closely related to the performance of the generator. At present, the silicon rectifier electrical excitation generator and permanent magnet generator are mostly used as vehicle generator in China, the losses of resistance in the former increased due to the existence of the excitation winding, thus the generator has low efficiency. Permanent magnet generator has the advantages of simple structure, low loss, but as its permanent magnet excitation magnetic field cannot be adjusted, it has the problem of the difficulty in voltage regulation. Particularly, when the output power of generator is greater than a certain value, to maintain the voltage stability would be even more difficult[1].

In this paper a new type of vehicle hybrid excitation synchronous generator was designed to solve the problem of electrical excitation generator and permanent magnet generator. It is a kind of promising vehicle generator, at same time the generator can make full use of the abundant rare earth resources in China, it is a novel vehicle generator which integrates motor, power electronics technology and new material technology, and it is able to adapt to the speed and load change in a wide range, while still maintaining a constant output voltage.

\section{Generator structure and working principle}

\subsection{Structure}

In this paper, a vehicle hybrid excitation synchronous generator which has rated power of $1 \mathrm{~kW}$, rated voltage of $400 \mathrm{~V}$ and rated speed of $3000 \mathrm{r} / \mathrm{min}$ is designed and studied, and it is shown in Fig.1. The generator consists of two parts: permanent magnet part and electric excitation part, they share a common winding called three-phase stator winding [2]. Two kinds of poles are staggered distributed on the rotor surface, one is permanent magnet pole, and the other is core pole. The circular field winding located in the middle of the stator core. Two parts of the rotor share a shaft, with a certain gap between them used as magnetic separation slot. At the same time, around the stator core there has a magnetic sleeve, which provides axial ring pathways for excitation winding. The permanent magnets produce radial field, and the electric excitation winding produce axial field and radial field both. Thus the two excitation sources produce synthetic magnetic field. The permanent flux starts from $\mathrm{N}$ pole in rotor surface, passes through the air gap and stator tooth and then goes into the stator iron core, passes through the stator yoke and another stator tooth, again through the air gap going into the rotor $\mathrm{S}$ pole, finally returns to the $\mathrm{N}$ pole, thus the magnetic circuit forms. Electric excitation flux goes through the

\footnotetext{
*Corresponding author: 1zs@fjut.edu.cn
} 
shaft, air gap, the stator tooth, yoke, the magnetic sleeve, stator teeth, yoke and air-gap in turn, thus forms a closed path. The magnitude of flux of electric field in the air gap is controlled by the excitation current [3], when field current remains constant, electrical excitation magnetic field produces a one-way direction rectangular magnetic flux in the air gap.

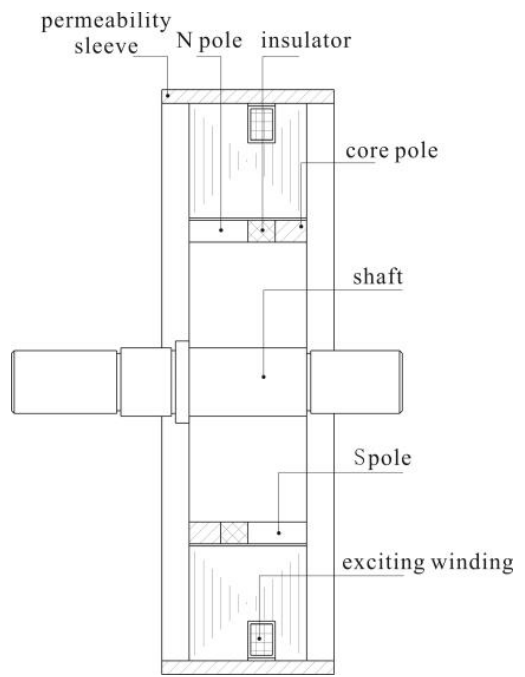

(a)Two-Dimension Structure

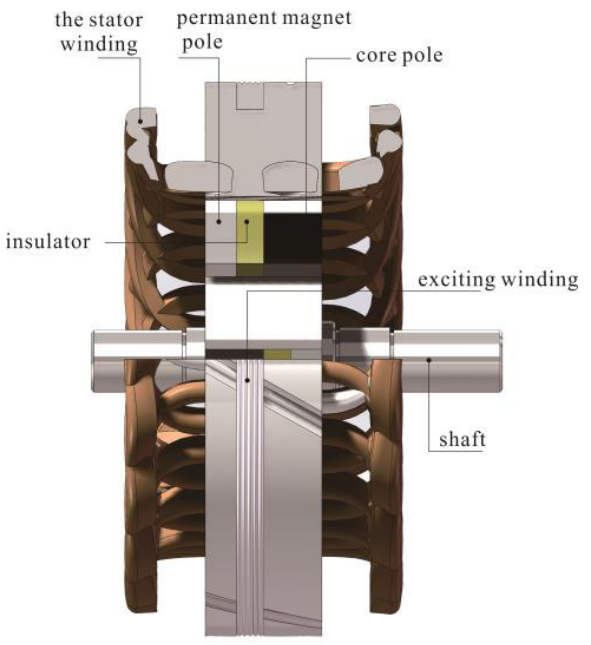

(b)Three-dimension structure

Fig.1. Hybrid excitation generator structure diagram.

\subsection{Electromagnetic optimum design}

The main technical indicators of vehicle HESG in this paper are as follows: rated power $1 \mathrm{~kW}$, rated voltage $240 \mathrm{~V}$, rated speed $3000 \mathrm{r} / \mathrm{min}$.

During design process, it should be noted that the load distribution between the permanent part and the electric excitation part determines the performance of the HESG. It requires that the HESG should have the voltage adjustment ability from no-load to rated load. And it can be seen from the operate principle of the HESG that we should ensure that the part of the magnetic circuit is in an unsaturated state when we design the HESG. While the HESG is with rated load, the electric excitation part plays a certain role in adding magnetism, and the permanent produces main magnetic field.

When the load is inductive load, the output voltage of the HESG is as follows:

$$
\begin{aligned}
U= & \sqrt{E_{d}^{2}+I_{N}^{2} X_{a q}^{2} \cos \psi_{N}-I_{N}^{2}\left(R_{1} \sin \varphi-X_{1} \cos \varphi\right)^{2}} \\
& -I_{N}\left(R_{1} \cos \varphi+X_{1} \sin \varphi\right)
\end{aligned}
$$

In above, $U$ is the output voltage of HESG; $E_{d}$ is the direct axis internal electric motive force. $\mathrm{I}_{\mathrm{N}}$ is the output current of HESG; $\mathrm{X}_{\mathrm{aq}}$ is the quadrature axis reactance of armature reaction; $\cos \psi_{N}$ is the internal power factor; $\mathrm{R}_{1}$ is the armature resistance; $\mathrm{X}_{1}$ is the armature leakage reactance and $\cos \varphi$ is the power factor.

It can be seen from formula (1) that we can increase the output voltage and reduce the voltage regulation by reducing the armature resistance $R_{1}$ and the armature leakage reactance $\mathrm{X}_{1}$.

The optimal design measures used in this paper is to keep the total number of turns per phase of the armature unchanged, meanwhile, to decrease the number of turns per coil. And finally use optimal measure to design the groove.

Combined with the above optimum design rule, using the special software ANSYS and the method for designing permanent magnet part and electric excitation part separately, we can optimize the design of HESG, and the results were shown in Table 1.

Table 1. Structure parameters of the prototype.

\begin{tabular}{cccc}
\hline Parameter & value & Parameter & value \\
\hline $\begin{array}{c}\text { Outer diameter of } \\
\text { the stator }\end{array}$ & $204 / \mathrm{mm}$ & $\begin{array}{c}\text { Outer diameter of } \\
\text { the rotor }\end{array}$ & $118.4 / \mathrm{mm}$ \\
\hline $\begin{array}{c}\text { Inner diameter of } \\
\text { the stator }\end{array}$ & $120 / \mathrm{mm}$ & $\begin{array}{c}\text { Inner diameter of } \\
\text { the rotor }\end{array}$ & $25 / \mathrm{mm}$ \\
\hline $\begin{array}{c}\text { The length of the } \\
\text { core }\end{array}$ & $50 / \mathrm{mm}$ & The air gap length & $0.8 / \mathrm{mm}$ \\
\hline $\begin{array}{c}\text { The thickness of } \\
\text { the magnet }\end{array}$ & $8 / \mathrm{mm}$ & Slot number & 36 \\
\hline $\begin{array}{c}\text { The width of the } \\
\text { magnet }\end{array}$ & $121 / \mathrm{mm}$ & Pole & 1 \\
\hline
\end{tabular}

\subsection{Chute stator core}

The generator stator core is chute designed, by using ANSYS Maxwell; we analyze the influence of different chute width on the generator voltage waveform sine distortion rate. The results are shown in Table 2.

Table 2. Voltage waveform sine distortion rate of different chute width.

\begin{tabular}{cccccc}
\hline The chute width & 0 & 0.25 & 0.5 & 0.75 & 1 \\
\hline $\begin{array}{c}\text { Linear voltage } \\
\text { waveform } \\
\text { distortion rate } \\
(\%)\end{array}$ & 1.762 & 1.248 & 1.126 & 0.893 & 0.674 \\
\hline $\begin{array}{c}\text { The chute width } \\
\text { Linear voltage } \\
\text { waveform } \\
\text { distortion rate } \\
(\%)\end{array}$ & 0.316 & 0.221 & 0.353 & 0.42 & \\
\hline & & 1.5 & 1.75 & 2 & \\
\hline
\end{tabular}


The table above shows that the stator chute can effectively reduce the magnitude of the sinusoidal output voltage waveform distortion rate, especially when the stator chutes width is 1.5 times the stator slot pitch, the generator voltage waveform sine distortion rate would be the minimum, and the sinusoidal of generator output voltage waveform would be the best. So, we chose the stator chute width as 1.5 times the stator slot pitch [4].

\subsection{Operation principle}

When the generator load reduces, the demagnetization effect of armature reaction would gradually weaken, it will lead to higher output voltage. The demagnetization current should be put into ring excitation winding current at this time, which makes the radial magnetic flux produced by the electric field at opposite direction of the radial air gap magnetic flux produced by permanent magnet, then the total air gap synthetic flux decreases, in this case the generator output voltage would reduce[4].

When the generator load increases, the demagnetization effect of armature reaction strengthen, results in the decrease of the output voltage, The enhancement magnetization current should be put into ring excitation winding current at this time, which makes the radial magnetic flux produced by the electric field at same direction of the radial air gap magnetic flux produced by permanent magnet, then the total air gap synthetic flux increases, in this case the generator output voltage would rise.

Above all, by adjusting the magnitude and direction of the hybrid excitation generator excitation current, we can change the syngas gap magnetic flux, so as to keep the output voltage constant when the load and speed change.

\section{HESG electromagnetic field}

\subsection{The field of permanent magnet}

Without considering the magnetic steel bonding, the magnetic field of permanent magnet of generator will be two dimension fields, a solution of the grid part of its area and no-load magnetic field distribution are shown in Fig.2. (a) and Fig.2. (b) respectively.

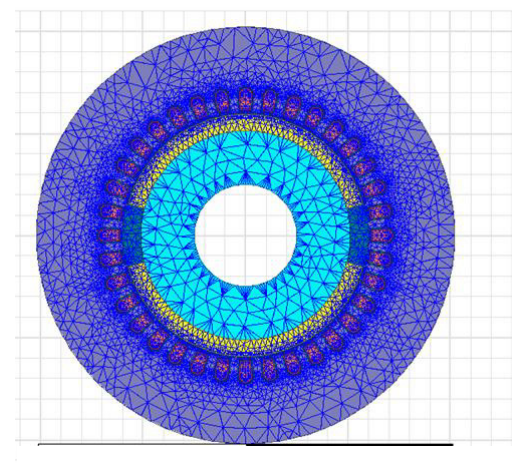

(a)the Grid Subdivision

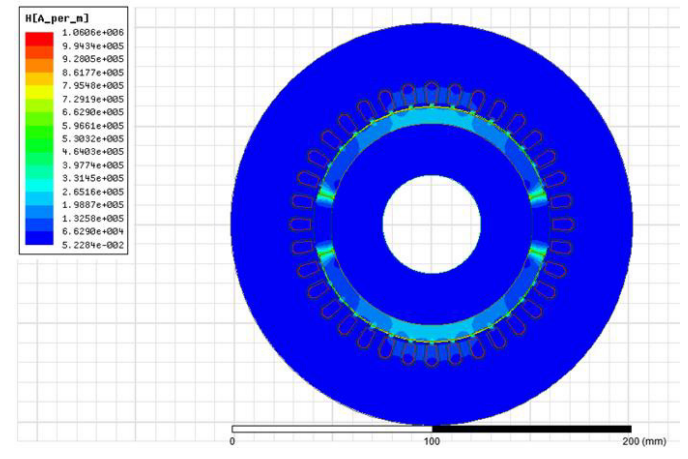

(b)Magnetic Field Distribution

Fig.2. Magnetic field distribution of permanent magnet.

\subsection{The field of electric excitation}

The grid subdivision and distribution of electromagnetic field of electric excitation are shown in Fig.3.(a) and Fig.3.(b) respectively. It can be seen from the diagram that the structure of the field of electric excitation is relatively complex, there exists both radial and axial magnetic flux in the solving region, and it is three dimension fields. The flux density in the shaft is higher, and prone to be saturation. At the same time, the field distribution of electric excitation along the axial is uneven; the field density on the boundary of the shaft surface between the slot and not slotted would be the maximum [5].

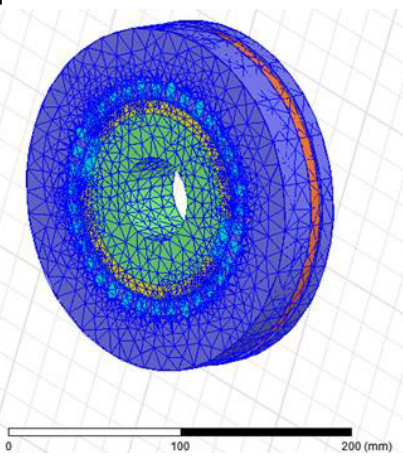

(a)The Grid Subdivision 


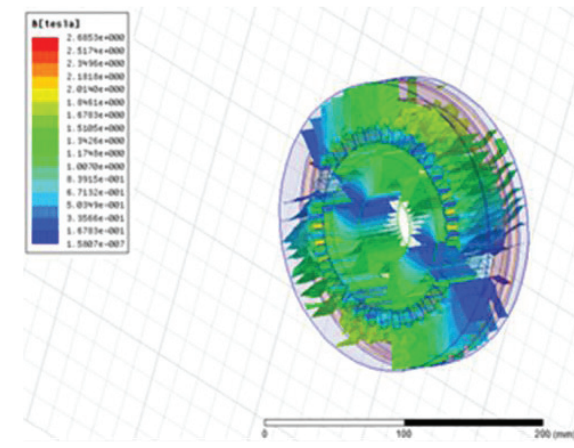

(b)Magnetic Field Distribution

Fig.3. Magnetic field distribution of electrical excitation.

\section{Automatic voltage regulation circuit design}

The permanent magnetic field and the electric field of the vehicle hybrid excitation generator synthesis are in the stator core. Because of its special structure, there are essential differences in .the principle of voltage control among hybrid excitation generator, traditional silicon rectifying generator and permanent magnet generator. In addition, due to the constantly changing of speed and load in the vehicle generators, to regulate the output voltage for maintaining voltage stability would be more difficult, the voltage regulating circuit is used to provide current to the hybrid excitation generator whose magnitude and direction can be adjusted[6]. When the voltage of the generator change with speed and load, the voltage regulating circuit can automatically adjust to keep the voltage of the generator at a given level and ensure the voltage regulation accuracy to a certain extent, by changing the magnitude and direction of excitation current, we can eliminate the influence of the armature reaction to make synthesis of magnetic field to be constant, and finally to meet the requirement of safe operation and normal generation. Its control principle is shown in Fig.4.

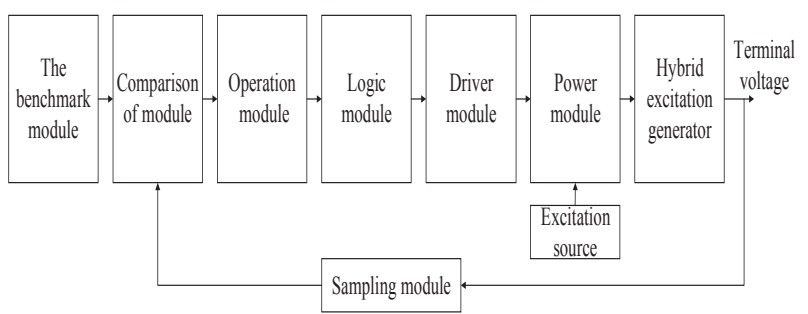

Fig.4. Hybrid excitation generator control principle diagram.

Voltage regulating control circuit designed in this paper consist of modules, such as the benchmark, comparison, operation, logical, drive, power and so on. when the output voltage of the generator is lower than the rating value of $400 \mathrm{~V}$, the control system through the control circuit and trigger circuit triggers the transistor so as to make the current flowing in the field winding is forward current[7], and then strengthen the excitation magnetic field, finally increase the output voltage of generator to ensure the output voltage of vehicle generator to reach to a specified value. When the output of the generator voltage is higher than the rating of $400 \mathrm{~V}$, the control circuit to trigger circuit triggered another pair transistor so as to make the current flowing in the field winding is reversal, and then weaken the excitation magnetic field, finally decrease the output voltage of generator, The cycle then repeats itself to ensure that the output voltage of generator remain unchanged in condition of wide range speed, variable load.

According to the voltage regulating circuit designed above, and then to make the experiment of HESM on the motor testing stand, the results of generator no-load test and load test are obtained as follows.

\subsection{No-load test}

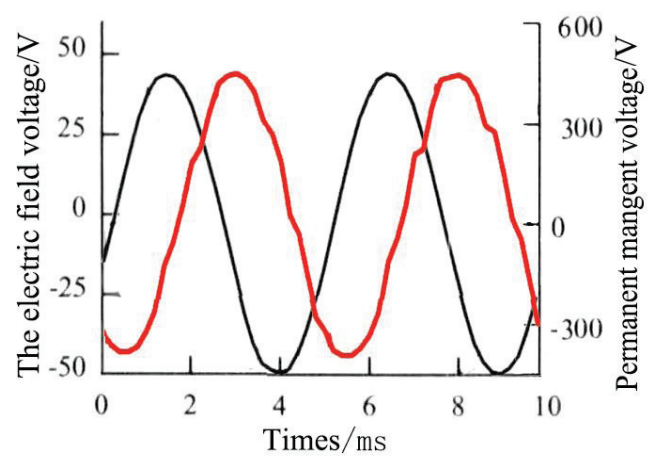

-Electric excitation voltage waveform

- Permanent magnet electric potential waveform

(a)Electric Field and Permanent Magnet Output Voltage Waveform

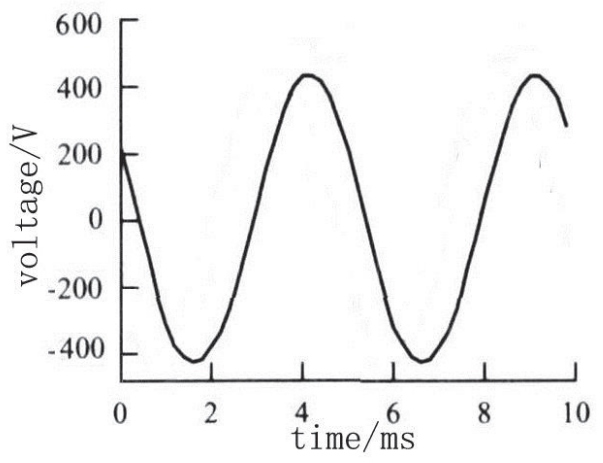

(b)Synthetic Voltage Waveform

Fig.5. Voltage waveform.

Generator operating in $3000 \mathrm{r} / \mathrm{min}$, no-load running under the action of electric excitation alone output voltage waveform, permanent magnet excitation alone under the action of the output voltage waveform and the above two parts respectively synthesized under the joint action of magnetic potential output voltage waveform are shown in Fig.5 (a). As it can be seen from Fig.5 (b).that 
the output voltage of generator is equal to the difference between the permanent magnet voltage and electrical excitation output voltage [8].

\subsection{The load test}

\subsubsection{External characteristic}

To convert generator output from $\mathrm{AC}$ to $\mathrm{DC}$, changing the generator speed and load at the same time [9], and keeping the output voltage maintaining in $400 \mathrm{~V}$, we can get the relationship between output voltage and exciting current, and the curve is shown in Fig.6.

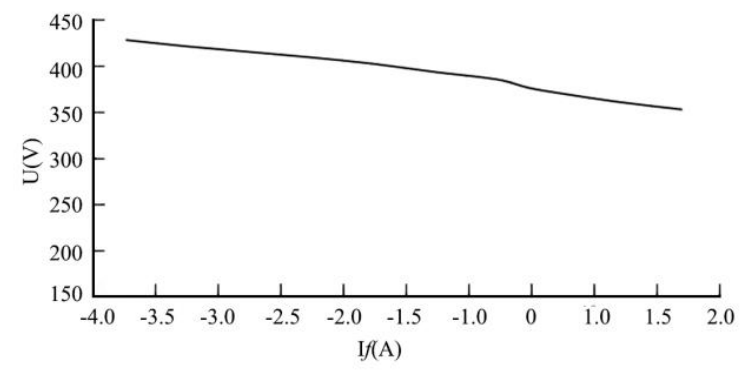

Fig.6. External characteristics.

Experimental result shows that: when the vehicle hybrid excitation generator load and speed change, the minimum output voltage is $352 \mathrm{~V}$, the maximum value is $428 \mathrm{~V}$, the voltage stabilizing circuit designed basically meets the design requirements, so we can obtain a stable voltage output in a wide range of load.

\subsubsection{The effects of generator parameters on the external characteristics}

Due to the changing of load and the stator current, the degree of saturation of the motor changes as while [10], thus affecting the generator armature reaction reactance, the inherent voltage regulation of the generator is:

$$
\Delta U \%=\frac{E_{0}-U}{U_{N}} \times 100 \%
$$

The inherent voltage regulation of hybrid excitation generator is defined as, when the electric field current is zero, the ratio of the armature voltage to the rated voltage. Parameters that affect the load voltage regulation are the stator resistance $R_{a}^{*}$, power factor $\cos \varphi$.

1) The effect of the stator resistance on the load characteristic

Fig. 7 shows the effect of stator resistance on external characteristic of generators, as can be seen in the Fig.7, the greater the stator resistance, the greater the inherent voltage regulation of the generator.

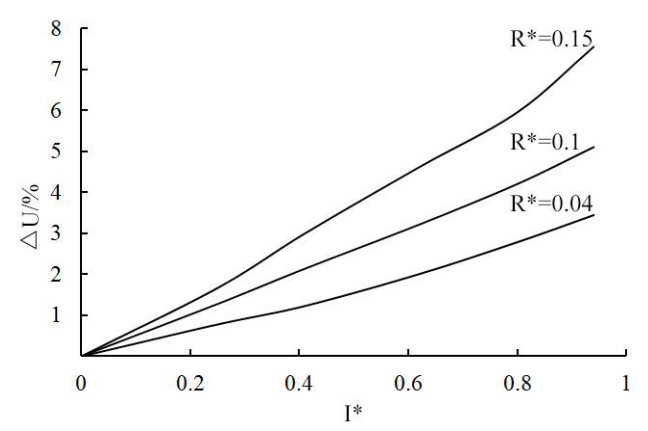

Fig.7. Effect of stator resistance on voltage regulation.

2) The effect of the power factor on external characteristic

From figure 8 , it can be seen that the greater the power factor, the smaller the inherent voltage change rate [9].

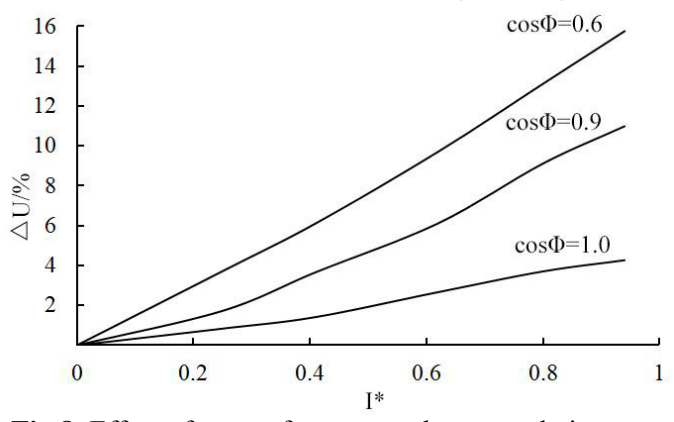

Fig.8. Effect of power factor on voltage regulation.

\subsection{Short circuit characteristic}

Short circuit characteristic refers to the relationship between short circuit current and excitation current when the three-phase synchronous generator is in short-circuits stability [11]. That the short circuit characteristics of hybrid excitation generator at different speeds result from the experiments are shown in Fig.9. Because the main flux does not equal zero when the excitation is zero, the short circuit characteristic curve does not pass through the origin [12]. It can be shown from the Fig.9 that the short circuit characteristic remains a straight line basically. It reflects that the magnetic circuit of the generator is in a state of unsaturation [13].

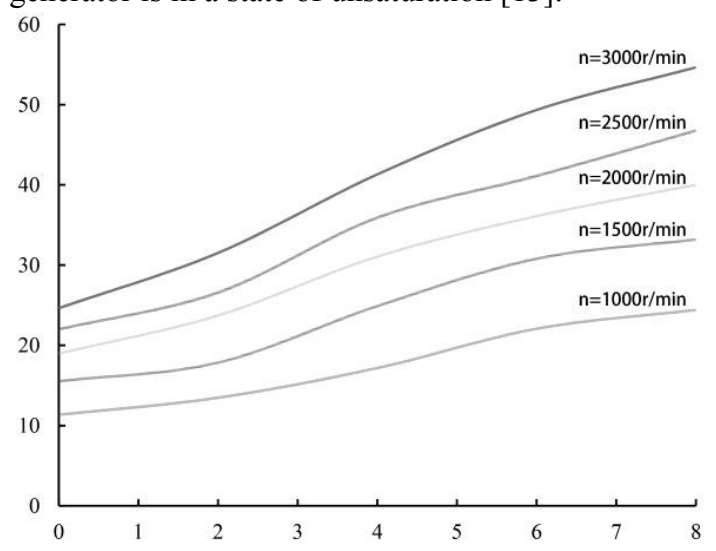

Fig.9. Short circuit characteristic under different speed. 


\section{Conclusion}

This paper designed a new type of vehicle hybrid excitation synchronous generator according to the principle of operation, analyzed the magnetic field of permanent magnetic parts and electrical excitation part, and finally an automatic voltage regulation system was designed applicable to hybrid excitation generator. In general, the new vehicle hybrid excitation generator has characteristics as follows:

First, the field winding embedded in the stator core, realizing the brushless excitation, as to improve the reliability of the generator operation [14].

Second, due to the electric excitation flux mainly through the core, adjusting the excitation current does not cause irreversible short circuit of permanent magnet, and there is no additional air gap in the magnetic circuit, so the power of field winding can be very small [15];

Third, a chute hybrid excitation generator stator core brings lower cogging torque, small output of the generator voltage waveform harmonic component and low waveform sine distortion rate.

Finally, owing to the permanent magnet used in the HESM, it can be made with high efficiency and lower density. Through the reasonable design of permanent magnet part and electric excitation part, it can meet the design requirements of different application [16].

Acknowledgments my work was supported by the Natural Science Foundation of Fujian Province of China. (Grant No. 2017J01667)

\section{References}

1. Ma Jianwei, Research on permanent magnet and electro-magnetic hybrid excitation constant voltage generator, (2008)
2. Lin Nan, Transaction of China electrotechnical society, 31, 19 (2016)

3. Xiao Li, Ge Fahua, Liu Tong, High speed high power density of generator to improve the design and implementation, 48, 18 (2015)

4. Jin Wanbing, Design and study on hybrid exciting permanent magnet generator and its control system, (2003)

5. Li Shenghu, An Rui, Xu Zhifeng, Dong Wangchao, Electric power automation, 35, 21 (2015)

6. Gong Jianfang, Large Electric machine and hydraulic turbine, 15, 17 (2008)

7. Shi Mingming, Zhou Bo, Wei Jiadan, Mao Yiran, Proceeding of the CSEE, 32, 1 (2012)

8. Wen Huihui, Control engineering of China, 21, 142 (2014)

9. Shen Xijun, Zhao Qiaoe, Xu Pengtao, Journal of Electric Power, 29, 28 (2014)

10. Zhang Zhuoran, Research on characteristics of novel hybrid excitation synchronous machines, (2008)

11. Feng Hao, Huo Zhihong, Hu Xuebin, Renewable energy resources, 33 , 1831 (2015)

12. Shen Jianxin, Miao Dongmin, Transactions of China Electrotechnical Society, 28, 2 (2013)

13. An Quntao, Sun Li, Sun Lizhi, Proceedings of the CSEE, 35, 5891 (2015)

14. Zhang Liguo, the Research and design on tangential structure hybrid excitation generator of magnetic multipled type for automobile, (2011)

15. Jian Zhijun, Micromotors, 46, 16 (2013)

16. Fu Xinghe, Zou Jibin, Qi Wenjuan, Journal of Southwest Jiao Tong Uniersity, 45, 99 (2010) 